\title{
Eine vereinfachte Mikromethode zur Bestimmung von Serumalbumin mit Hilfe von Bromkresolgrün \\ $\cdot 1$
}

\author{
Von H. Schirardin und J. Ney \\ Laboratoire de Patbologie Générale, Clinique Médicale A, Centre Hospitalo-Universitaire, (Directeur: Prof. J. Warter) \\ Strasbourg (France) und Service de Biologie Médicale, Centre Hospitalier, (Chef de Service: Dr. J. Ney) Sarreguemines (France)
}

(Eingegangen am 16. Februar 1972)

Eine einfache und zuverlässige Albuminbestimmungsmethode wird beschrieben. Ihr Prinzip beruht auf einer Farbkomplexbildung zwischen Albumin und Bromkresolgrün. Die Methodik ist einfach und erlaubt eine schnelle Ausführung. Sie benötigt nur wenige Mikroliter Serum oder Plasma. Die Reagenzien sind lange haltbar und die Gestehungskosten sind unbedeutend. Die erhaltenen Ergebnisse stimmen gut mit der Sulfitfällungsmethode und der Celluloseacetatelektrophorese überein. Die Präzision ist groß und die hohe Empfindlichkeit erlaubt die Benutzung einfacher Einstrahlfilterphotometer. Das Verfahren eignet sich deshalb hervorragend für klinische Routinearbeit.

\section{Determination of serum albumin by a simplified dye-binding micromethod using bromcresolgreen}

A simple and well-tried test for serum albumin is described. It is based on the formation of a dye-combination between albumin and bromcresolgreen. The technique, which is of extreme simplicity, can be carried out in very little time and requires only a few microlitres of serum or plasma. The preparation of the reagents is easy, their storage very satisfactory and they are not expensive. The results obtained fit in well with the salt fractionation techniques with sodium sulfite and electrophoresis with cellulose acetate. It is very accurate; owing to its great sensitivity, the results can be read even on very simple filter photometers. The data indicate that the proposed technique will prove very useful, especially in daily hospital routine.

\section{Determination de la Serumalbumine a l'aide d'une micrometbode simplifiée utilisant le vert de Bromecresol}

Une méthode simple et éprouvée de détermination de l'albumine sérique est décrite. Son principe est basé sur la formation d'un complexe coloré entre l'albumine et le vert de bromecrésol. La technique, d'une simplicité extrème et d'exécution rapide, ne nécessite que quelques microlitres de sérum ou de plasma. La préparation des réactifs est facile, leur conservation très bonne et leur prix de revient très modéré. Les résultats obtenus s'accordent bien avec la technique de précipitation au sulfite de sodium et l'électrophorese sur acétate de cellulose. La précision en est grande; sa forte sensibilité permet la lecture sur des photomètres à filtres de conception modeste.

Toutes ces données nous font admettre, que la technique proposée peut rendre de grands services surtout en routine clinique.

Serumalbumin wurde früher nach Aussalzung der Globuline mit Natriumsulfat oder besser Natriumsulfit photometrisch mit Biuret- oder FolIn-Reagenz bestimmt $(1-3)$. Eine Vereinfachung wat später die Extraktion des Albumins mit einer trichloressig- oder perchlorsauren Äthanollösung und Bestimmung durch UV-Absorption (4-8).

Die Elektrophorese gilt wegen ihrer Zuverlässigkeit noch immer als Standardmethode für die Eiweißbestimmung im Blutplasma (9-11). Neuerdings kann Serumalbumin durch Immunpräzipitation spezifisch bestimmt werden (12-17).

$\mathrm{Zu}$ den schnellsten und bequemsten Bestimmungsmethoden gehören ohne Zweifel jene, welche sich die Bildung eines Farbkomplexes zwischen Albumin und $\mathrm{pH}$-Indikatoren $\mathrm{zu}$ eigen machen.

Die hierfür zur Zeit gebțäuchlichsten Indikatoren sind Methylorange $(18,19)$, Carboxy-2-hydroxy-4'azo-benzol (20-22), Bromkresolpurpur (23) und Bromkresolgrün (24).

Die Farbkomplex-Bildung mit Methylorange hat sich als nicht sehr spezifisch für Albumin erwiesen, die mit Carboxy-2-hydroxy-4'-azobenzol wird durch höhere Bilirubinkonzentrationen und verschiedene Arzneimittel gestört.
Neuere Arbeiten (25-30) haben gezeigt, daß Bromkresolgrün einen sehr spezifischen Albumin-Farbkomplex bildet und sich deshalb gut zur quantitativen Albuminbestimmung eignet.

Wir haben versucht, die bestmöglichen Reaktionsbedingungen und Verhältnisse zwischen Albumin und diesem Indikator herauszufinden und eine einfache spezifische und quantitative Albuminbestimmung auszuarbeiten.

\section{Material und Methoden \\ Geräte}

Spektralphotometer oder Filtexphotometer zur Messung bei $625 \mathrm{~nm}$. Mikropipetten von. $10 \mu 1$ oder $15 \mu \mathrm{l}$ Inhalt.

Reagenzien

1. Essigsäure 99-100proz. zur Analyse.

2. Natronlauge $1 \mathrm{Mol} / \mathrm{l}$ (Titrisol).

3. Polyhydroxyäthylenlauryläther (Brij 35).

4. Bromkresolgrün, Indikator (Merck, Nr. 8121).

Herstellung der Lösungen

Essigsäure, $1 \mathrm{Mol} / 1$

$60 \mathrm{~g}$ Essigsäure mit destillịertem Wasser ad $1000 \mathrm{ml}$ mischen.

Acetat=Brij-Puffer, $0,05 \mathrm{Mol} / \mathrm{l}, \mathrm{pH} \mathrm{4,2}$

$50 \mathrm{ml}$ Essigsäure $1 \mathrm{Mol} / 1$ und $13,2 \mathrm{ml}$ Natronlauge $1 \mathrm{Mol} / 1 \mathrm{mit}$ etwa $500 \mathrm{ml}$ dest. Wasser mischen, nach Zugabe von $0,85 \mathrm{~g}$ Brij 35 
und vollständigem Auflösen mit dest. Wasser auf $1000 \mathrm{ml}$ auffüllen. Im Plastikbehälter ist der Puffer bei $+4^{\circ} \mathrm{C}$ über ein Jahr haltbar.

\section{Bromkeresolgrïn-Stammlösung 1,2 mMol/1}

$0,838 \mathrm{~g}$ Bromkresolgrün in $20 \mathrm{ml}$ Natronlauge $0,1 \mathrm{Mol} / 1$ auflösen (am besten die Lösung $24 \mathrm{~h}$ in einem 1 l-Meßkolben stehen lassen und von Zeit zu Zeit schütteln). Dann mit dest. Wasser auf $1000 \mathrm{ml}$ auffüllen. In dunkler Flasche bei $+4^{\circ} \mathrm{C}$ ist diese Stammlösung über 6 Monate hinaus haltbar.

\section{Gebrauchsfarbreagenz}

Genau $85 \mathrm{ml}$ Bromkresolgrün-Stammlösung mit Acetat-BrijPuffer ad $1000 \mathrm{ml}$ verdünnen. Diese Lösung ist 2 Monate bei Raumtemperatur haltbar.

\section{Humanalbumin ${ }^{1}$ ) Standarllösung}

Humanalbumin krist. trocken wird in dest. Wasser gelöst. Bei Zusatz von Natriumazid, $1 \mathrm{~g} / 1$ ist die Lösung 6 Monate haltbar.

\section{Stabilität der Lösungen}

Acetat-Brij-Puffer und Bromkresolgrün-Stammlösung wurden über ein Jahr bei $+4^{\circ} \mathrm{C}$ aufbewahrt. Gebrauchsfarbreagenz, das aus gelagerten und frischen Lösungen hergestellt wurde, zeigte keine Unterschiede in der Farbintensität bei Albuminzusatz. Gebrauchsfarbreagenzlösung wurde ferner 3 Monate lang teils bei Raumtemperatur, teils bei $+4^{\circ} \mathrm{C}$ aufbewahrt und alle 2 bis 3 Tage zur Albuminbestimmung desselben Humankontrollserums (jedesmal frisch in Lösung gebracht) herangezogen. Nach 3-monatiger Lagerung der Gebrauchslösung bei $+4^{\circ} \mathrm{C}$ erhielten wir die gleichen Extinktionswerte wie am Tage der Herstellung. Dagegen hielt sich das bei Raumtemperatur $\left(22^{\circ} \mathrm{C}\right)$ aufbewahrte Gebrauchsreagenz nur 2 Monate.

\section{Arbeitsweise}

Man verteilt zuerst das Gebrauchsfarbreagenz, gewöhnlich 4 oder $5 \mathrm{ml}$, in Glas- oder Polyäthylen-Röhrchen, gibt dann $10 \mu \mathrm{l}$ bzw. $15 \mu \mathrm{l}$ Serum hinzu, (am besten durch Einblasen mit der Mikropipette in das Farbreagenz), mischt nochmals gut und photometriert.

Die Extinktion kann im Spektralphotometer bei $625 \mathrm{~nm}$ oder mit jedem anderen Filterphotometer gemessen werden.

Die Messung erfolgt bei Raumtemperatur in Küvétten oder MeßRöhrchen von $1 \mathrm{~cm}$ Schichtdicke gegen den Leerwert, bestehend aus Gebrauchslösung. Bei jeder Meßreihe wird zur Auswertung ein geeigneter Humanalbumin-Standard mitgeführt $(29,31)$.

\section{Berechnung}

Bei Verwendung eines Standards ergibt sich der Albumingehalt aus der Gleichung:

$$
\frac{E_{A}}{E_{S}} \cdot S=g / 1 \text { Albumin }
$$

$\mathrm{E}_{\mathrm{A}}$ : Extinktion der Analyse

Es : Extinktion des Standards

$\mathrm{S}$ : Konzentration des Standards in $\mathrm{g} / \mathrm{l}$.

\section{Standardlösungen}

Humanalbumin ${ }^{1}$ ) $4 \mathrm{mal} \mathrm{krist.} \mathrm{wurde} \mathrm{zur} \mathrm{Herstellung} \mathrm{der} \mathrm{Standard-}$ lösungen verwendet. Die Gehaltsbestimmung erfolgte durch Messung der UV-Absorption bei $280 \mathrm{~nm}$ (32) und Stickstoffbestimmung mittels Nesslerisierung (33).

Der Gehalt des Präparates an Albumin wurde mit 81 und 81,2\% bestimmt. Das unter üblichen Trocknungsbedingungen behandelte Albumin erlaubte uns den restlichen Anteil von 19\% fast ausschließlich dem Feuchtigkeitsgehalt zuzuschreiben.

Ein im Handel erhältlicher lyophylisierter Humanalbumin-Stan$\mathrm{dard}^{2}$ ) mit einer Gehaltsangabe von $80 \mathrm{~g} / 1$ wurde durch 4 ver-

1) Nutritional Biochemicals Co., Cleveland, Ohio 44128 USA.

2) Dade Division, Amer. Hosp. Supply Corp. Miami, Fla. 33152 (USA).

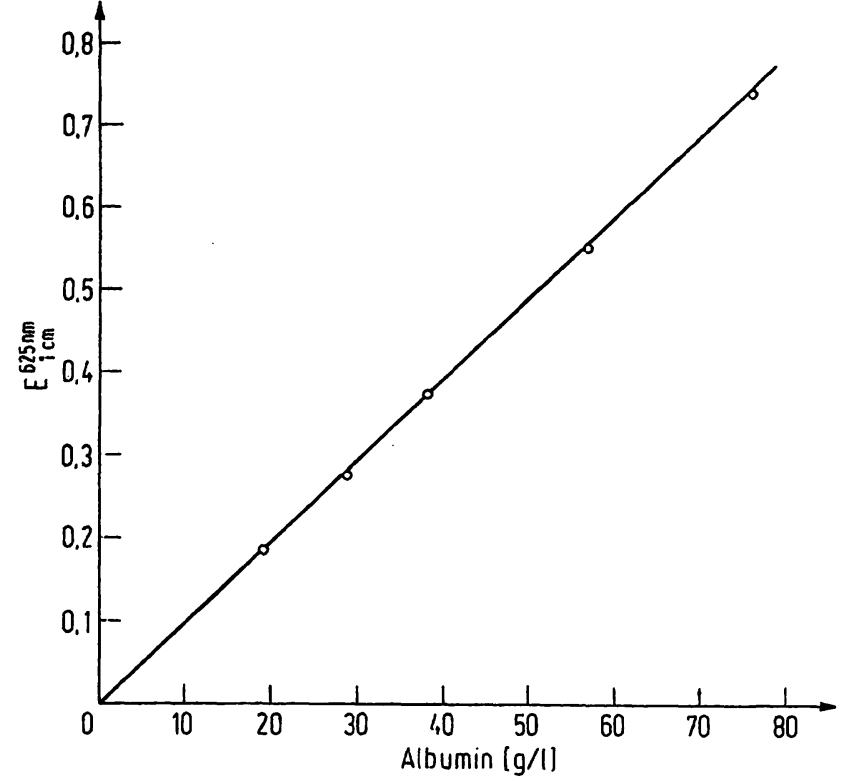

Abb. 1

Extinktionsänderung bei steigender Humanalbumin ${ }^{2}$-konzentration Ansatz: Humanalbuminlösung $20 \mu \mathrm{l}$; Gebrauchsfarbreagenz $8 \mathrm{ml}$ gemessen im Spektralphotometer Beckman D. U.

schiedene Verfahren getestet. Wir erhielten in der Folge: durch Stickstoffbestimmung (33) $80,5 \mathrm{~g} / 1$; mit der Biuret-Methode (34) $80,4 \mathrm{~g} / 1$; durch Messung der UV-Absorption bei $280 \mathrm{~nm}$ (32) $79,2 \mathrm{~g} / \mathrm{l}$ und mit der Bromkresolgrün-Methode 79,0 g/l.

Die Ubereinstimmung einer Humanalbumin-Standardlösung zu $80 \mathrm{~g} / \mathrm{l}$ mit einem Human-Kontrollserum ${ }^{3}$ ) zu 38,0 g/l wurde geprüft. Die ermittelte Konzentration des Kontrollserums war mit $38,6 \mathrm{~g} / \mathrm{l}$ dem angegebenen Wert fast gleich.

Zur Prüfung der Gültigkeit des LAMBert-BeERschen Gesetzes wurde eine aus kristallinem Humanalbumin ${ }^{1}$ ) hergestellte Standardlösung eingesetzt. Die unter Berücksichtigung der angegebenen Albumingehalte aufgestellte Kurve verläuft linear bis $75 \mathrm{~g} / \mathrm{l}$, gemessen im Spektralphotometer Beckman D. U. (Abb. 1).

Normbereich der Methode

Der Normalbereich $\overrightarrow{\mathbf{x}} \pm 2 \mathrm{~s}$ wurde anhand von 30 Blutspenderseren ermittelt. Die Albuminstreubreite erstreckt sich von 37 bis $46 \mathrm{~g} / \mathrm{l}$, während die entsprechenden Gesamteiweißwerte zwischen 59 und $71 \mathrm{~g} / 1$ liegen $(34,35)$. Wir legten besonderen Wert darauf, Seren von Leberkranken oder nicht nüchternen Spendern auszuschließen.

Stabilität des Farbkomplexes

Diese Studie wurde mit einem Serumpool durchgeführt. $\mathrm{Zu}$ diesem Zweck wurde die Extinktion des Farbkomplexes in Abhängigkeit von der Zeit verfolgt und jedesmal der Durchschnittswert von 24 Einzelbestimmungen desselben Serumpools vermerkt.

Wie aus Tabelle 1 hervorgeht, hat die Farbintensität gleich nach Zugabe der Probe zum Gebrauchsfarbreagenz ihren Höchstwert erreicht. Der Abfall der Färbung ist bedeutungslos bis $6 \mathrm{~h}$, ja sogar $24 \mathrm{~h}$ nach Ausführung des Ansatzes.

\section{Anmerkung}

Die Benutzung eines einfachen Filterphotometers ist dank der hohen Empfindlichkeit der Methode für die Routinepraxis vollauf ausreichend.

Die im Mikroliterbereich liegenden Probevolumina verlangen höchste Anforderungen an die benutzten Mikropipetten. Die besten Resultate erzielten wir ausschließlich mit Uberflußpipetten („Blow-out“-Pipetten) ${ }^{4}$ ).

3) Hyland Div. Travenol Lab. Inc. Los Angeles, Calif. 90039 (USA).

4) Misco Berkeley 10. California (USA). 
Tab. 1

Zeitlicher Verlauf der Extinktionswerte eines Serumpool-Gebrauchsreagenz-Ansatzes

\begin{tabular}{|c|c|c|}
\hline Stunden & Extinktionswerte & . \\
\hline 0 & 0,585 & \\
\hline 1 & 0,585 & \\
\hline 2 & 0,585 & \\
\hline 3 & 0,580 & \\
\hline 4 & 0,580 & \\
\hline 5 & 0,580 & \\
\hline 6 & 0,580 & \\
\hline 8 & 0,575 & \\
\hline 24 & 0,570 & \\
\hline
\end{tabular}

\section{Ausarbeitung optimaler Meßbedingungen}

\section{Wahl der Pufferlösung}

Bei vergleichenden Albuminbestimmungen stellten wir bei Verwendung von Acetatpuffer die höchsten Extinktionswerte fest. Succinat- und Formiatpuffer ergaben gering niedrigere Werte (29). Die erhaltenen Extinktionen bei Verwendung von Citratpuffer lagen um fast $30 \%$ niedriger als diejenigen in Acetatpuffer (Tab. 2), (27, 28). Phosphatpuffer bei pH 7 hingegen eignet sich sehr schlecht für Albuminbestimmungen. Das Gebrauchsreagenz ist dunkelblau und gibt bei Albuminzusatz negative Extinktionswerte (25).

\section{Einfluß der Ionenstärke}

Bei einer Reihenuntersuchung mittels Gebrauchslösung abnehmender Molaritäten verzeichneten wir zuerst eine Zunahme der Extinktionswerte für Pufferlösungen von $0,2 \mathrm{Mol} / 1$ bis $0,05 \mathrm{Mol} / \mathrm{l}$, dann einen leichten Rückgang der Extinktion für die $0,025 \mathrm{Mol} / 1$ Pufferlösung (Abb. 2). Daher haben wir die Acetatgebrauchslösung 0,05 Mol/l den anderen vorgezogen.

\section{$\mathrm{pH}-$ Wert}

Wir setzten Gebrauchslösungen mit verschiedenen $\mathrm{pH}$-Werten von 3,5 bis 5,4 zur Albuminbestimmung ein. Die Extinktionen nahmen bis zu einem Höchstwert bei $\mathrm{pH} 4,2 \mathrm{zu}$, um dann gleichmäßig abzunehmen (Abb. 3).

Tab. 2

Abhängigkeit der Extinktionswerte von Gebrauchsfarbreagenzien, hergestellt mit verschiedenen Puffersystemen

Gemessen wurden die Extinktionen im Spektralphotometer Beckman D. U. nach Zugabe von $20 \mu \mathrm{l}$ Humanserum (38. g/l Albumin) $\mathrm{zu} 8 \mathrm{ml}$ Gebrauchsfarbreagenz, hergestellt nach den angegebenen Daten

\begin{tabular}{|c|c|c|c|c|}
\hline $\begin{array}{c}\text { Angewandte } \\
\text { Puffersysteme }\end{array}$ & $\begin{array}{l}\text { Konzen- } \\
\text { tration } \\
(\mathrm{Mol} / \mathrm{l})\end{array}$ & $\mathrm{pH}$ & $\begin{array}{c}\text { Extinktions- } \\
\text { werte } \\
625 \mathrm{~nm} \\
1 \mathrm{~cm}\end{array}$ & Ausbeute \% \\
\hline $\begin{array}{l}\text { Essigsäure/ } \\
\quad \text { Natronlauge }\end{array}$ & 0,05 & 4,2 & 0,365 & 100 \\
\hline $\begin{array}{c}\text { Bernsteinsäure/ } \\
\text { Natronlauge }\end{array}$ & 0,05 & 4,2 & 0,345 & 94,5 \\
\hline $\begin{array}{l}\text { Ameisensäure/ } \\
\text { Natronlauge }\end{array}$ & 0,05 & 4,2 & 0,320 & 90 \\
\hline $\begin{array}{l}\text { Natriumcitrat/ } \\
\text { Salzsäure }\end{array}$ & 0,05 & 4,2 & 0,265 & 72,5 \\
\hline
\end{tabular}

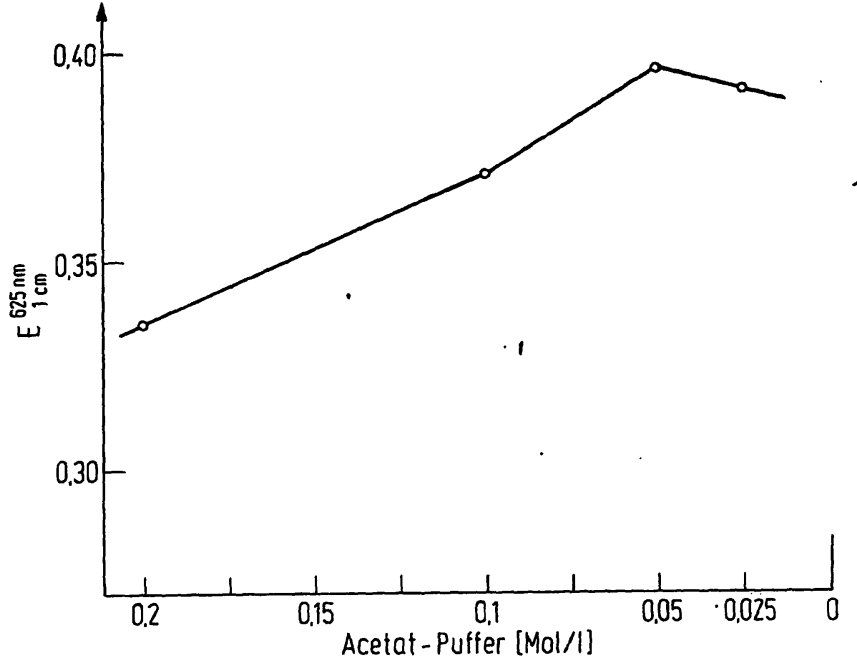

Abb. 2

Abhängigkeit der Extinktionswerte von Gebrauchsfarbreagenzien mit abnehmender Puffer-Konzentration Gemessen wurden die Extinktionen im Spektralphotometer Beckman D. U. nach Zugabe von $20 \mu$ I Humanserum ( 38 g/l Albumin) $2 u \quad 8 \mathrm{~m}$ Gebrauchsfarbstoffreagenz, hergestellt mit Brij-Acetatpuffer $\mathrm{pH} 4,2$ verschiedener Konzentration und Bromkresolgrün-Stammlösung
$1,2 \mathrm{mMol} / 1$

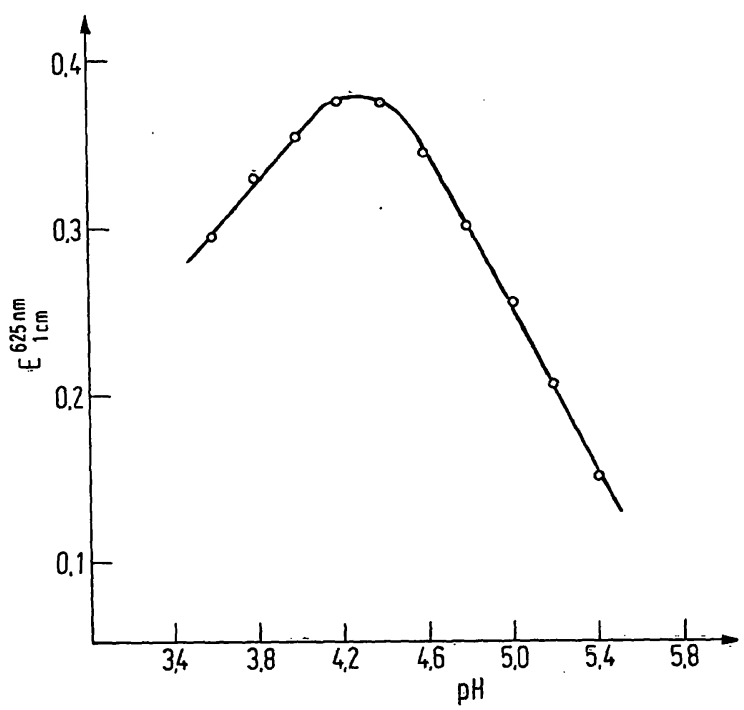

Abb. 3

Abhängigkeit der Extinktionswerte von Gebrauchsfarbreagenzien mit verschiedenen $\mathrm{pH}$ Werten

Gemessen wurden die Extinktionen im Spektralphotometer Beckman D. U. nach Zugabe von $20 \mu \mathrm{l}$ Humanserum ( $38 \mathrm{~g} / \mathrm{l}$ Albumin) $\mathrm{zu} 8 \mathrm{~m}$ Gebrauchsfarbreagenz hergestellt mit Brij-Acetatpuffer $0.05 \mathrm{Mol} / \mathrm{l}$ $(\mathrm{pH}$ variiert) und Bromkresolgrün-Stammlösung $1,2 \mathrm{mMol} / \mathrm{l}$

\section{Bromkresolgrün-Konzentration}

Durch Variation der Konzentration von Bromkresolgrün im Gebrauchsreagenz von 6 bis $120 \mu \mathrm{Mol} / 1$ stellten wir fest, $\mathrm{da} \beta$ die Extinktionswerte und die Linearität bei einem Gehalt von $102 \mu \mathrm{Mol} / 1$ Bromkresolgrün im Gebrauchsfarbreagenz optimal sind. (Abb. 4).

\section{Einfluß oberflächenaktiver Substanzen}

Die Zugabe oberflächenaktiver Substanzen zur Pufferlösung und somit zum Gebrauchsfarbreagenz wirkt stabilisierend und verhütet Trübungen im Farbreagenzserumgemisch. Versuche mit verschiedenen Wasserstoffionen-Konzentrationen unseres Farbreagenzes ohne Zusatz oberflächenaktiver Substanzen haben uns ge- 


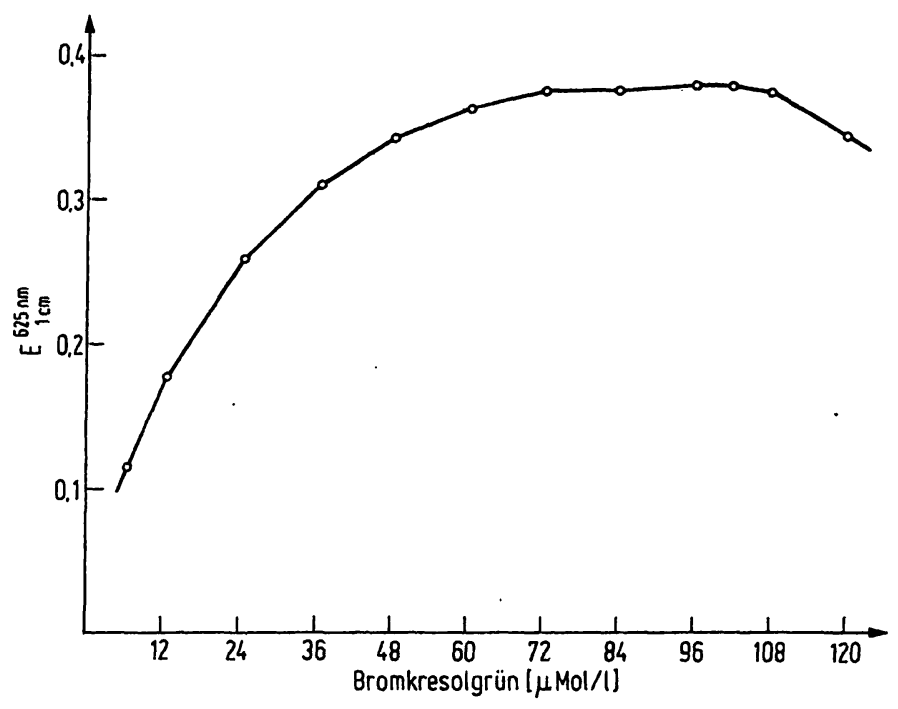

Abb. 4

Abhängigkeit der Extinktionswerte von Gebrauchsfarbreagenzien mit zunehmenden Bromkresolgrün-Konzentrationen

Gemessen wurden die Extinktionen im Spektralphotometer Beckman D. U. nach Zugabe von $20 \mu \mathrm{l}$ Humanserum $(3,8 \mathrm{~g} / \mathrm{l}$ Albumin) zu $8 \mathrm{~m}$

G. U. nach Zugabe von 20 l Humanserum $(3,8 \mathrm{~g} / \mathrm{l}$ Albumin) zu $8 \mathrm{~m}$
pH 4,2 und Bromkresolgrün-Stammlösung $1,2 \mathrm{mMol} / \mathrm{l}$ (variiert)

zeigt, daß die Proteine nach Zugabe der Serumprobe zum Teil ausfallen, und zwar im Bereich von $\mathrm{pH} 3,6$ und 4,8. Verschiedene oberflächenaktive Substanzen wurden nacheinander in unseren Acetatpuffer eingesetzt, so z. B. Brij 35, Tween 20, Teepol, Natriumlaurylsulfat und Cetrimid.

Brij 35. (Polyhydroxyäthylenlauryläther) und Tween 20 (Polyhydroxyäthylensorbitanmonolaurat) haben sich am besten geeignet. Teepol, Natriumlaurylsulfat und Cetrimid (Hexadecyltrimethylammoniumbromid) ergaben mit Acetatpuffer und Bromkresolgrün ein Farbreagenz mit dunklem Leerwert. Schließlich haben wir Brij 35 dem Tween 20 vorgezogen, denn letzteres ist dickflüssig und deswegen schwer zu handhaben.

Die Konzentration von Brij 35 im Farbreagenz, die wir verwenden, ist kritisch. Sie beträgt $0,85 \mathrm{~g} / \mathrm{l}$ in der Pufferlösung und $0,78 \mathrm{~g} / 1$ in der Gebrauchslösung. Der optimale Extinktionswert jedoch wurde mit 1,05 g/1 Brij 35 in der Gebrauchslösung nach Albuminzugabe ermittelt (Abb. 5), steht aber nicht im Einklang mit dem Linearitätsoptimum. Die Gebrauchslösung erfährt eine deutliche Farbänderung von blau-grün bis gelb mit der Zunahme an Brij 35. Die Annahme eines Ausgleichsfarbtones der Gebrauchslösung, welcher mit $0,78 \mathrm{~g} / \mathrm{l}$ erreicht wurde, ersparte uns daher den Verlust der Linearität bei nur geringer Einbuße an Empfindlichkeit.

\section{Zuverlässigkeitsprüfung der Methode}

\section{Einfluß von Globulinfraktionen}

Mehrere Handelspräparate, $\alpha$-, $\beta$ - und $\gamma$-Globulin ${ }^{1}$ ) sowie eine $\gamma$-Myelomfraktion in $\mathrm{NaCl}$-Lösung gebracht, dienten uns zur Interferenzprüfung. Nach der Bestimmung des Reinheitsgrades dieser Lösungen mittels Elektrophorese wurde bei bekannter Arbeitsweise die

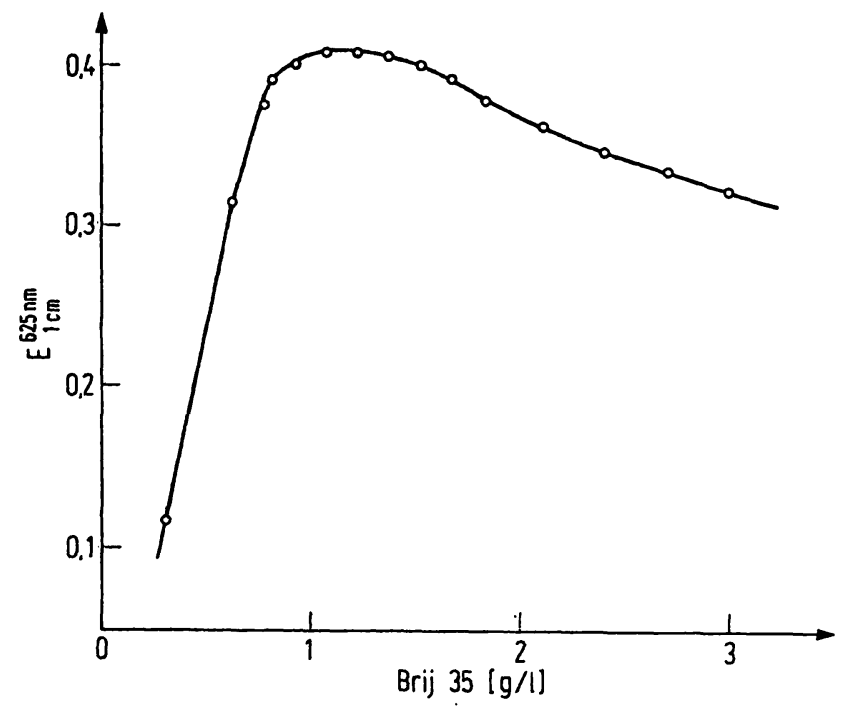

Abb. 5

Abhängigkeit der Extinktionswerte von Gebrauchsfarbreagenzien mit ansteigenden Brij 35-Konzentrationen

Gemessen wurden die Extinktionen im Spektralphotometer Beckman D. U. nach Zugabe von $20 \mu \mathrm{l}$ Humanserum ( $38 \mathrm{~g} / 1$ Albumin) zu $8 \mathrm{~m}$ Gebrauchsfrabreagenz, hergestellt mit Brij 35-(variiert) Acetatpuffer $0,05 \mathrm{Mol} / 1 \mathrm{pH} 4,2$ und Bromkresolgrün-Stammlösung 1,2 mMol/1
(1,

Gegenprobe mit der Bromkresolgrün-Methode vorgenommen. Die Ergebnisse haben wir in der Tabelle 3 zusammengefaßt. Das eingesetzte Human- $\alpha$ - und $\beta$ Globulin war mit Albumin verunreinigt. Die Human- $\gamma-$ Globulinfraktionen dagegen waren albuminfrei. Diese Feststellung konnte jedesmal durch beide Prüfungsmethoden bestätigt werden.

In einem weiteren Versuch trennten wir drei normale Seren an Celluloseacetat im Makroverfahren und benutzten als Farbreagenz unsere Gebrauchslösung. Es zeigte sich, daß letztere ausschließlich die Albuminfraktion stark blau anfärbte.

\section{Einfluß von Bilirubin}

Serum wurde mit ansteigenden Mengen Bilirubin angereichert. Bis $\mathrm{zu}$ einem Gehalt von $400 \mathrm{mg} / \mathrm{l}$ ergab sich keine Interferenz. Demnach kann Albumin un-

Tab. 3

Prüfung der Interferenz durch Eiweißfraktionen unterschiedlichen Reinheitsgrades

$\begin{array}{cccc}\text { Eiweiß-Fraktion } & \begin{array}{c}\text { Konzentration } \\ \text { g/l }\end{array} & \begin{array}{c}\text { Elektrophorese } \\ \text { Albumin g/l }\end{array} & \begin{array}{c}\text { Bromkresol- } \\ \text { grün-Methode } \\ \text { Albumin } g / l\end{array} \\ & & \end{array}$

\begin{tabular}{lccc}
$\begin{array}{l}\text { Human- } \\
\alpha-G l o b u l i n\end{array}$ & 2,1 & 2,2 \\
$\begin{array}{l}\text { Fraktion IV/I } \\
\text { Human- } \\
\beta \text {-Globulin } \\
\text { Fraktion III })\end{array}$ & 10 & 6,9 & 6,0 \\
$\begin{array}{l}\text { Human- } \\
\gamma \text {-Globulin } \\
\text { Fraktion II })\end{array}$ & 15 & rein & 0 \\
$\begin{array}{l}\text { Human- } \\
\gamma \text {-Globulin } \\
(M y e l o m)\end{array}$ & 20 & rein & 0 \\
\hline
\end{tabular}


cingeschränkt in ikterischen Seren bestimmt werden. Sollte aber der Bilirubingehalt $400 \mathrm{mg} / 1$ übersteigen, ist eine Verdünnung der Probe zu empfehlen.

\section{Arzneimittel-Einfluß}

Der Einfluß einiger Arzneistoffe, welche während ihres Transportes an Albumin gebunden sind, wurden getestet. So wurde Serum mit Sulfanilamid $10 \mathrm{~g} / \mathrm{l}$, Tromexan (Bis-3,3'-(4-hydroxycumarinyl)-essigsäureäthylester) $6 \mathrm{~g} / \mathrm{l}$, Pindione (2-Phenyl-1,3-indandion) 0,8 g/l, Sintrom (3-[ $\alpha$-(4-Nitrophenyl)- $\beta$-acetyläthyl]-4-hydroxycumarin) $0,1 \mathrm{~g} / 1$ und Aspirin (Acetylsalicylsäure) $1 \mathrm{bis}$ $5 \mathrm{~g} / \mathrm{l}$ versetzt und eine Stunde lang zur Inkubation beiseite gestellt. Diese Proben sowie die Vergleichsseren wurden dann zur Albuminbestimmung herangezogen. Keines dieser Medikamente hatte den geringsten Einfluß auf die Albuminbestimmung, obwohl die eingesetzten Mengen die übliche 24-h-Dosis weitaus überschritten.

Vergleichsuntersuchungen $z$ wischen Serum und Plasma

Serum und Plasma von demselben Patienten wurden frisch und nach längerer Aufbewahrung bei $+4^{\circ} \mathrm{C}$ in Zeitabständen untersucht. Es ergab sich absolut keine Differenz in den gefundenen Werten zwischen Serum und Plasma. Beide können gleichwertig zur Albuminbestimmung mit der Bromkresolgrün-Methode verwendet werden.

\section{Lipämische Seren}

Stark getrübte Seren können, bedingt durch den sehr hohen Verdünnungsgrad unserer Arbeitsweise (1:400) ohne weiteres benutzt werden. Bei abnorm hohen Trübungen stellt sich die Notwendigkeit, das Serum $1+1$ mit Wasser zu verdünnen oder nur die Hälfte der Probe in den Ansatz zu bringen. Gegebenenfalls kann auch ein Leerwert mitgeführt werden.

\section{Hämolytische Seren}

Normale und mit Hämoglobin angereicherte Seren gleicher Herkunft dienten uns zur Albuminbestimmung. Bei schwacher Hämolyse wurde ein unbedeutender Anstieg des Albuminwertes verzeichnet, jedoch konnten wir bei starker Hämolyse (5 g/l Hämoglobin) eine Steigerung des Albumins von nahezu 5\% wahrnehmen. Aufgrund dieser Feststellung sind hämolytische Seren oder Plasma möglichst zu vermeiden.

\section{Richtigkeit}

Zur Prüfung der Richtigkeit wurden Seren eines gemischten Krankenhaus-Kollektivs untersucht, welches Albuminkonzentrationen von 18 bis $55 \mathrm{~g} / 1$ mit entsprechenden Albumin-Globulin-Quotienten zwischen 0,4 und 1,9 aufwies.

Die Vergleichsanalysen wurden mit der hier beschriebenen Bromkresolgrün-Methode sowie der SulfitBiuret-Methode $(2,3)$ und der Celluloseacetatmikroelektrophorese (9) ausgeführt.
Die daraus errechneten Korrelationen bestätigen, daß unsere Methode sowohl niedrige als auch hohe Albuminkonzentrationen richtig erfaßt.

Die Berechnung der Korrelationskoeffizienten und der Regressionsgeraden erfolgte entsprechend den in Tabelle 4 angegebenen Gleichungen (Tab. 4) (36). Die graphische Darstellung der Wertepaare ist in Abbildung 6 und 7 veranschaulicht.

Tab. 4

Berechnung von Korrelationskoeffizienten und Regressionsgeraden

\begin{tabular}{|c|c|c|}
\hline & $\begin{array}{c}\text { Vergleich } \\
\text { Elektrophorese (x) } \\
\text { u. Bromkresol- } \\
\text { grün }(y)\end{array}$ & $\begin{array}{l}\text { Vergleich } \\
\text { Sulfit-Biuret (x) } \\
\text { u. Bromkresol- } \\
\text { grün (y) }\end{array}$ \\
\hline n & 220 & 162 \\
\hline$\overline{\mathbf{x}}=\frac{\Sigma_{\mathbf{x}}}{\mathrm{n}}(\mathrm{g} / \mathrm{l})$ & 36,77 & 35,81 \\
\hline$S_{x}=\Sigma(x-\bar{x})^{2}$ & 10532,32 & 9809,29 \\
\hline$s_{x}^{2}=\frac{s_{x}}{n-1}$ & 48,09 & 60,93 \\
\hline$s_{\mathbf{x}}$ & 6,93 & 7,81 \\
\hline $\bar{y}=\frac{\Sigma y}{n}(g / l)$ & 36,64 & 35,97 \\
\hline$S_{y}=\Sigma(y-\bar{y})^{2}$ & 8528,37 & 7732,48 \\
\hline$s_{y}^{2}=\frac{s_{y}}{n-1}$ & 38,94 & 48,03 \\
\hline sy & 6,24 & 6,93 \\
\hline$S_{x y}=\Sigma(x-\bar{x}) \cdot(y-\bar{y})$ & 8438,53 & 8258,73 \\
\hline$s_{y \cdot x}^{2}=s_{y}^{2}\left(1-r^{2}\right) \frac{n-1}{n-2}$ & 8,11 & 4,87 \\
\hline$s_{x \cdot y}^{2}=s_{y}^{2}\left(1-r^{2}\right) \frac{n-1}{n-2}$ & 10,01 & 6,18 \\
\hline $\mathrm{r}$ : Korrelationskoeffizient & 0,890 & 0,948 \\
\hline $\begin{array}{l}\text { Formeln der } \\
\text { Regressionsgeraden }\end{array}$ & $\begin{array}{l}Y=7,18+0,80 x \\
X=0,51+0,99 y\end{array}$ & $\begin{array}{l}Y=5,83+0,84 x \\
X=-2,61+1,07 y\end{array}$ \\
\hline
\end{tabular}

\section{Präzision}

Die Kenngrößen Mittelwert, Standardabweichung und Variationskoeffizient sowie die Vergleichswerte der Sulfit-Biuret-Methode sind aus Tabelle 5 ersichtlich $(37,38)$.

Diesen Daten liegt eine 50-fache Albuminbestimmung eines normalen Serumpools mit $35 \mathrm{~g} / 1$ zugrundé.

Tab. 5

Präzisionskontrolle der Bromkresolgrün-Methode, verglichen mit der Sulfit-Biuretmethode

Bromkresolgrün-Methode Sulfit-Biuretmethode

\begin{tabular}{lcc}
\hline $\mathrm{n}$ & 50 & 50 \\
$\overline{\mathrm{x}}(\mathrm{g} / \mathrm{l})$ & 35 & 35 \\
$\mathrm{~s}(\mathrm{~g} / \mathrm{l})$ & $\pm 0,70$ & $\pm 1,14$ \\
$100 \cdot \frac{\mathrm{s}}{\bar{x}}[\%]$ & 2 & 3,3 \\
\hline
\end{tabular}

\section{Empfindlichkeit}

Die Empfindlichkeit wird bei praktischen Ausführungen gleich zwei Standardabweichungen (2s) gesetzt (39). Sie beträgt $1,4 \mathrm{~g} / 1$ für unsere Bromkresolgrün-Methode, und als Vergleich 2,3 g/l für die SulfitBiuret-Methode. 


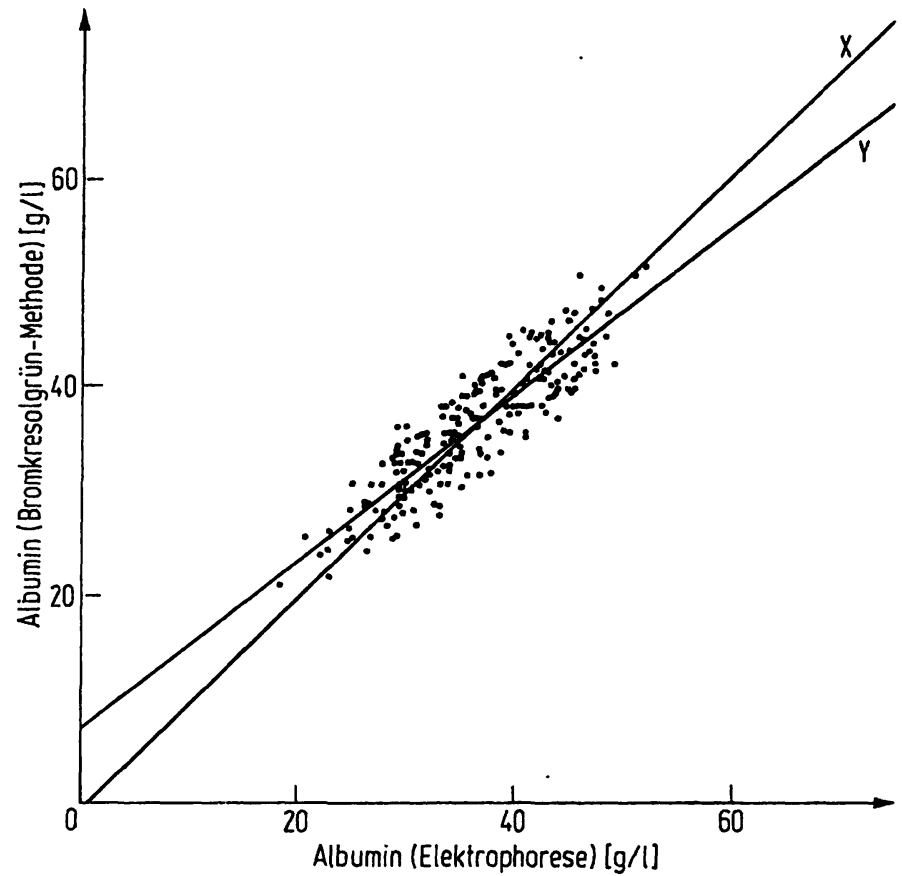

Abb. 6

Punkte-Korrelationsdiagramm zwischen der Celluloseacetat-Mikroelektrophorese und der hier beschriebenen Bromkresolgrün-Methode

\section{Diskussion}

Die von uns beschriebene Albuminbestimmungsmethode beruht auf dem bekannten Prinzip der Farbstoffkomplexbildung. Dabei diente uns Bromkresolgrün als Farbstoff. Bei der Ausarbeitung des Verfahrens legten wir größten Wert auf die Wahl der Pufferlösung, deren Ionenstärke und Wasserstoffkonzentration; wir konnten mit Acetatpuffer $0,05 \mathrm{Mol} / \mathrm{l}$ bei pH 4,2 die größten Extinktionswerte erhalten. Auch die Bromkresolgrün- und Brij 35-Konzentrationen in der Gebrauchslösung wurden durch Versuche festgesetzt. Sie betragen $102 \mu \mathrm{Mol} / 1$ für Bromkresolgrün und $0,78 \mathrm{~g} / \mathrm{l}$ für Brij 35. Ferner stellten wir fest, daß Pufferkonzentration, pH-Wert, Konzentrationen von Farbstoff- und oberflächenaktiver Substanz in enger Ver-

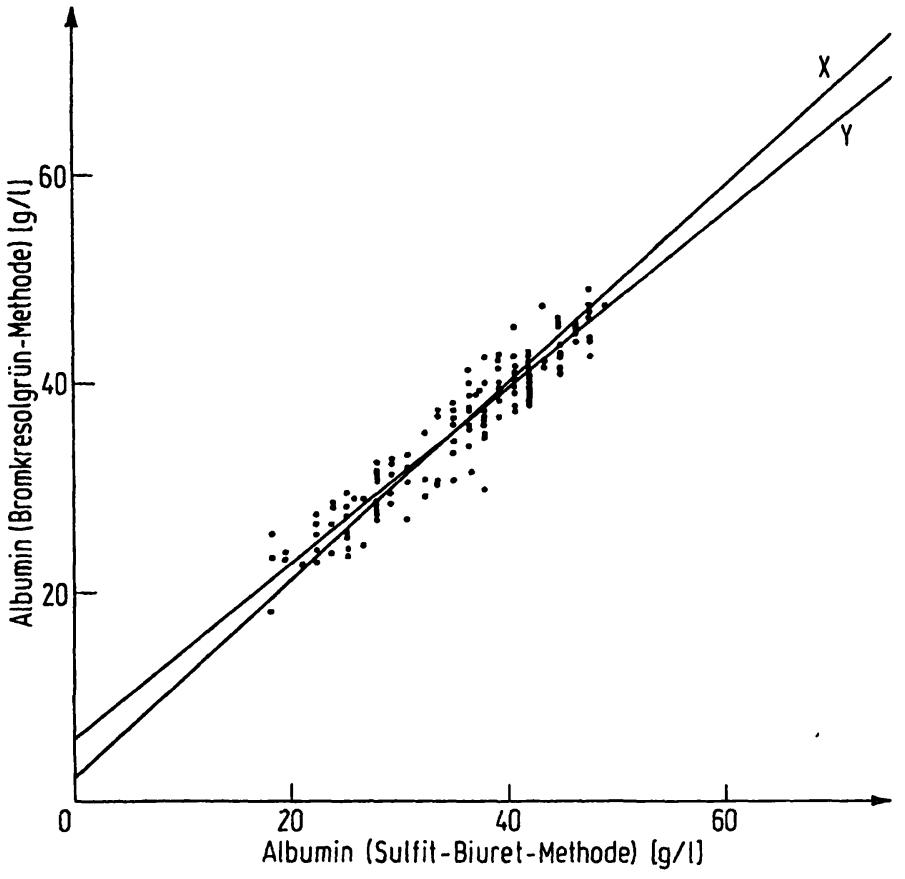

Abb. 7 Punkte-Korrelationsdiagramm zwischen der Sulfit-Biuret-Methode
und der hier beschriebenen Bromkresolgrün-Methode

bindung zueinander stehen und zur Aufrechterhaltung der Linearität beitragen.

Sollte aber einer dieser Faktoren weit von den oben angegebenen Daten abweichen, so wird ein Rückgang der Empfindlichkeit mit Linearitätsverlust verzeichnet.

Das Eintreten schwerwiegender Störeffekte, hervorgerufen durch normale oder fremde Begleitsubstanzen konnte nicht festgestellt werden. Wir möchten lediglich darauf hinweisen, stark hämolytische Seren $\mathrm{zu}$ vermeiden und stark lipämische Seren vor der Albuminbestimmung $z u$ verdünnen. Die statistische Prüfung bestätigte uns durch Vergleiche mit anderen Verfahren, daß unsere Methode mit den bisher üblichen völlig gleichwertig ist.

\section{Literatur}

1. Campbell, W. R. \& Hanna, M. I. (1937), J. Biol. Chem. 119 15-33. - 2. Cohn, C \& Wolfson, W. Q. (1948), J. Lab. Clin. Med. 33, 367-370. - 3. Wolfson, W. Q., Cohn, C., Calvary, E. \& Tromas, E. M. (1948), J. Lab. Clin. Med. 33, 1276-1288. 4. WAdDEL, W. J. (1956), J. Lab. Clin. Med. 48, 311-314. 5. BeNdixen, G. (1957), Nord. Med. Tidkr. (Stockholm) 58 , 1487-1489. -6. TOMBs, M. P., Souter, F. \& MAC LAGAN, N. F. (1959), Biochem. J. 73, 167-171. - 7. MURpiY, J. B. \& KIES, M. W. (1960), Biochim. Biophys. Acta 45, 382-384. - 8. GANDOLFI, E. \& FABRINI, G. (1966), Ital. J. Biochem. 15, 244-249. 9. Instruction Manual R. M.-I-M.-3 (1965), p. 13, Beckman Instruments Inc., Fullerton, Cf. USA. - 10. KAPLAN, A \& SAvory, J. (1965), Clin. Chem. 11, 937-942. - 11. Luxton, G. C. (1968), Can. J. Med. Technol. 30, 83-101. - 12. Schultze, H. E. \& Schwrck, H. G. (1959), Clin. Chim. Acta 4, 15-25. 13. Oudin, J. (1949), C. R. Acad. Sci. 228, 1890-1892. - 14. Feinberg, J. G. (1957), Int. Arch. Allergy Appl. Immunol. 11, 129-152. - 15. HaYward, B. J. \& Augustin, R. (1957), Int.
Arch. Allergy Appl. Immunol. 11, 192-205. - 16. Mancini, G., Carbonara, A. O. \& Heremans, J. F. (1965), Immunochemistry 2, 235-254. - 17. Laurell, C. B. (1966), Anal. Biochem. 15, 45-52. - 18. Bracken, J. S. \& Klotz, J. M. (1953), Amer. J. Clin. Pathol. 23, 1055-1058. - 19. Keyser, J. W. (1961), Clin. Chim. Acta 6, 445-447. - 20. Ru'rstern, D. D., Ingenito, E. F. \& Reynozds, W. E. (1954), J. Clin. Invest. 33, 211-221. 21. Bohuon, C. \& Leboeuf, M. (1964), Ann. Biol. Clin. (Paris) 22, 133-141. - 22. Arvan, D. A. \& Ritz, A. (1969), Clin. Chim. Acta 26, 505-516. - 23. LouderaACK, A. Mealey, E. H. \& TaYLOR, N. A. (1968), Clin. Chem. 14, 793-794. - 24. Rodkey, F. L. (1964), Arch. Biochem. Biophys. 108, 510-513. - 25. RoDKEY, F. L. (1965), Clin. Chem. 11, 478-487. - 26. Bartholomew, R. J. \& Delaney, A. M. (1966), Proc. Aust. Ass. Clin. Biochem. 1, 214-219. - 27. Hernandez, O., Murray, L. \& Dumas, B. (1967), Clin. Chem. 13, 701. - 28. Dow, D. \& Pinto, P. V. C. (1969), Clin. Chem. 15, 1006-1008. - 29. Doumas, B. T., Watson, W. A. \& Brggs, H. G. (1971), Clin. Chim. Acta 31, 87-96. - 


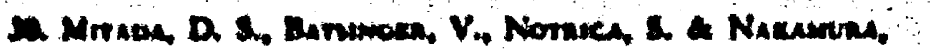

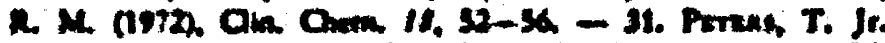

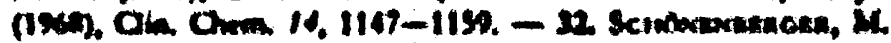

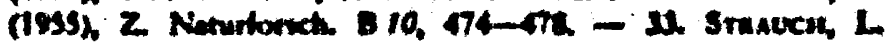

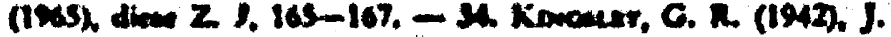

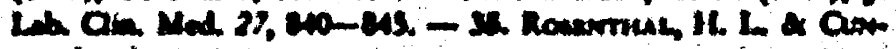

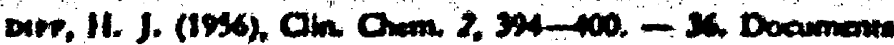

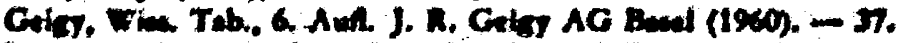

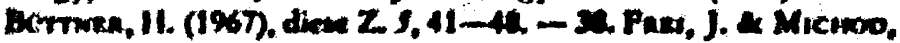
1. (1971), Sctweth. Med. Wocheneche. 101, 24-20. - 37. Ricum:

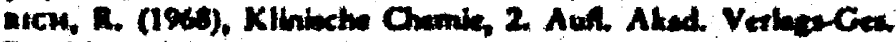
Frankfary/Minin.
Dr. H. Sehimedia Leb. Oln Mad. A Hopital Cini 67. Straboure Bunkin Prunce) 